\title{
Mitigation of pathogens and marine biotoxins contamination in shellfish
}

\author{
P. Fajardo ${ }^{1}$, M. Atanassova ${ }^{1}$, J. Cotterill ${ }^{2}$, T. Wontner-Smith ${ }^{2}$, \\ J. Vieites ${ }^{1} \&$ A. Cabado ${ }^{1}$ \\ ${ }^{I}$ ANFACO-CECOPESCA, Vigo, Spain \\ ${ }^{2}$ FERA, York, $U K$
}

\begin{abstract}
The EU FP7 funded project "Bio-engineered micro Encapsulation of Active agents Delivered to Shellfish (BEADS)" is focused on mitigating the impact of marine biotoxins (ASP/DSP), microbial contamination (bacteria/norovirus) and the parasitic protozoan Bonamia ostreae on shellfish aquaculture.

Purpose: to develop probiotic diets and a microencapsulated delivery system in the digestive tract of shellfish to improve depuration.

Feeding experiments were performed to identify the optimum size of alginate microcapsules, testing three different sizes and colours containing nondegradable fluorescent dye microbeads.

Oysters and mussels were placed in tanks containing filtered seawater. Three sizes of microcapsules were mixed and shellfish were fed for 3 hours. Shellfish were removed during the feeding period at $0.5,1,2$ and 3 hours and dissected. Any capsules remaining in the water and in the digestive organs of shellfish were extracted and measured using a fluoroskan analyzer.

A higher concentration of the smaller capsules was found in the digestive gland, indicating that the smaller capsules were preferentially ingested. The ingestion increased during the three-hour period. Fluorescent beads of different colours embedded in alginate capsules were observed bound to the mucus string.

Mussels were more efficient than oysters in incorporating alginate beads that were observed by light microscopy as intact in the digestive gland of mussels and oysters during feeding period. Broken alginate beads were found in faeces.

During passage through the intestine, active agents embedded by alginate capsules are released into the digestive gland providing a useful tool to transport active agents.
\end{abstract}


Keywords: microcapsules, alginate, microbeads, shellfish, microbial contamination, Bonamia ostrae, virus, marine biotoxins, detoxification, depuration.

\section{Introduction}

Bivalve production has increased from 5.3 million tonnes in 1990 to 14.6 million tonnes in 2010. This increase is due to the fast growth of aquaculture production, which increased from 3.3 million tonnes, equivalent to $62 \%$ of total bivalve production, to 12.9 million tonnes in $2010,88 \%$ of total [1]. Nutritious properties of molluscs have been thoroughly studied and it is well known that they constitute an essential part of a healthy and balanced diet; thus, shellfish consumption has expanded considerably. However, the safety of seafood and especially shellfish, which are sometimes consumed raw, is of great concern for consumers and health authorities.

The development of shellfish aquaculture, mussels, oysters, clams, cockles, or scallops, among others, has to face many problems such as economic losses, due to Harmful Algae Blooms (HAB), shellfish diseases, especially those related to parasites and microbial contaminations. A significant issue affecting aquaculture production is the development of toxic episodes that induce the contamination of shellfish through naturally occurring potent algal toxins produced by toxic phytoplankton. The main toxins affecting European waters include: paralytic shellfish poisoning (PSP), amnesic shellfish poisoning (ASP) and lipophilic toxins, previously called diarrheic shellfish poisoning (DSP), for review [2]. Other risks that hamper the development of shellfish-based industries include microbiological contamination with faecal material, bacteria or virus derived from human or animal sources and the spreading of parasites and diseases (e.g. Bonamia ostrea causing bonamiasis in the flat oyster) [3].

The EU FP7 funded project "Bio-engineered micro Encapsulation of Active agents Delivered to Shellfish (BEADS)" www.projectbeads.eu, is focused on mitigating the impact of marine biotoxins (ASP/DSP), microbial contamination (bacteria/norovirus) and the parasitic protozoan Bonamia ostreae on shellfish aquaculture. The consortium is formed by small and medium-size enterprises (SMEs) and several research institutions. The objective of the project is to develop probiotic diets and a microencapsulated delivery system in the digestive tract of shellfish to improve depuration efficiency and toxin degradation.

In order to investigate the use of administered micro-capsules as an aid to effective and efficient depuration of pathogens, bacteria, viruses, parasites and degradation of microalgal toxins some protocols for shellfish feeding microencapsulated probiotics diets were developed. 


\section{Material and methods}

\subsection{Biological samples}

Oysters (Ostrea edulis) and mussels (Mytilus galloprovincialis) were obtained locally from Galicia, NW of Spain.

\subsection{Microencapsulation}

Research related to the EC FP6 Collective Research Project Spies-Detox, has demonstrated that it is possible to successfully microencapsulate marine compounds using sodium alginate as matrix. Alginate capsules were proposed for encapsulation since alginate has good mucoadhesive properties and forms soft gel micro-spheres, or beads, that had previously been found to be ingested and digestible by fish and shellfish. Non-degradable styrene maleic anhydride (SMA) microbeads with fluorescent dyes were prepared by FERA using alginate as a carrier. Microencapsulation was carried out using a Nisco Var J30 encapsulation unit equipped with a $500 \mu \mathrm{m}$ nozzle.

\subsection{Feeding experiments}

Feeding experiments with mussels and oysters were performed to identify the optimum size of alginate microcapsules containing non-degradable SMA microbeads. Three sizes of alginate beads were tested, each size containing SMA microbeads with a different fluorescent dye. A number of 30 mussels or 24 oysters were placed in a tank containing $40 \mathrm{~L}$ of filtered sea water at a temperature of $15^{\circ} \mathrm{C}$. After an acclimatizing period of 24 hours, the shellfish were fed with two concentrations of alginate beads, 100 and $10 \mathrm{mg} / \mathrm{L}$, over a $3 \mathrm{~h}$ period (feeding period). A lyophilized commercial microalgae was included to stimulate shellfish feeding. Equal quantities of the three sizes of microcapsules were mixed together and shellfish were fed for a period of 3 hours. After this period, shellfish were placed into tanks containing clean water and were kept under these conditions for 42 hours. Shellfish were removed during the feeding period at $0.5,1,2$ and 3 hours and dissected. Also samples were analyzed after 24 and $48 \mathrm{~h}$ (depuration period) to analyze the clearance of the beads.

\subsection{Fluorescence analyses}

In order to dissolve the alginate beads, the digestive gland and gills of oysters and the digestive gland and the rest of the body of mussels were removed separately. Alginate capsules were macerated with $4 \mathrm{ml}$ of $1 \mathrm{M}$ sodium citrate using a mortar or an ultra turrex to dissolve the alginate. To dissolve the fluorescent SMA beads, $4 \mathrm{ml}$ of acetone was added and the mixture was macerated and centrifuged. To analyze fluorescence of microbeads, $250 \mu 1$ of the supernatant was transferred to the wells of a 96 well black plate and the fluorescence at each excitation/emission wavelength of the three types of dye was measured in a fluorescent plate reader. These experiments were performed 
in triplicate. Any capsules remaining in the water and in the digestive organs of shellfish were extracted and measured using the fluoroskan analyzer.

Spiked samples were prepared by incubating both, mussels and oysters samples, with $200 \mu$ of each type of microencapsulated bead separately and all together. The alginate and the fluorescent SMA beads were dissolved using the procedure outlined above. Control samples were used in order to estimate the shellfish auto-fluorescence.

Interferences in the fluorescence between the different dyes were also taken into account when data were processed.

\section{Results}

First we proved that alginate capsules are dissolved by macerating with $1 \mathrm{M}$ sodium citrate using a mortar or an ultra turrex to release the microbeads which can then be analyzed. Fig 1 shows the SMA microbeads (A) contained in alginate capsules and (B) after dissolving the alginate capsule with $1 \mathrm{M}$ sodium citrate.

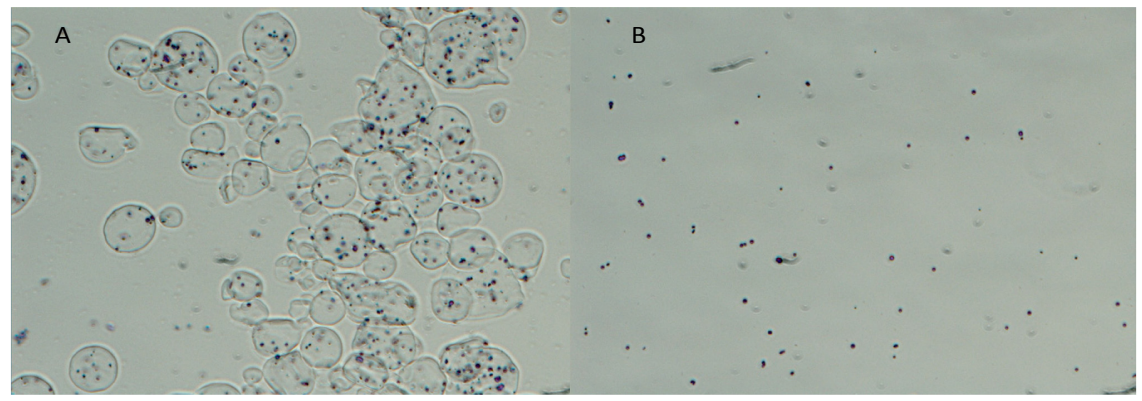

Figure 1: $\quad$ SMA microbeads (A) contained in alginate capsules and (B) after dissolving the alginate capsule with $1 \mathrm{M}$ sodium citrate.

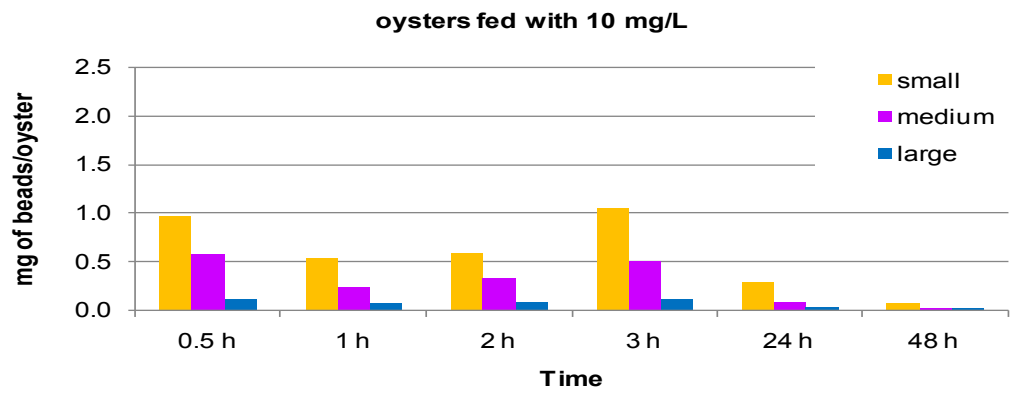

Figure 2: Concentration of fluorescent beads in the digestive gland of oysters fed with $10 \mathrm{mg} / \mathrm{L}$ of a mixture of alginate capsules of different sizes. 
Mixed microcapsules were found in digestive gland, gills or in rest of the body of the shellfish. However, a higher concentration of the smaller capsules was found in mussels and oyster indicating that the smaller capsules were preferentially ingested (Figs 2-5). The ingestion of capsules increased progressively during the three-hour feeding period. After this period the shellfish were moved to $40 \mathrm{~L}$ tanks with fresh seawater and the elimination of microbeads from the digestive gland started. However after $48 \mathrm{~h}$ mussels and oysters still retained some fluorescent microbeads.

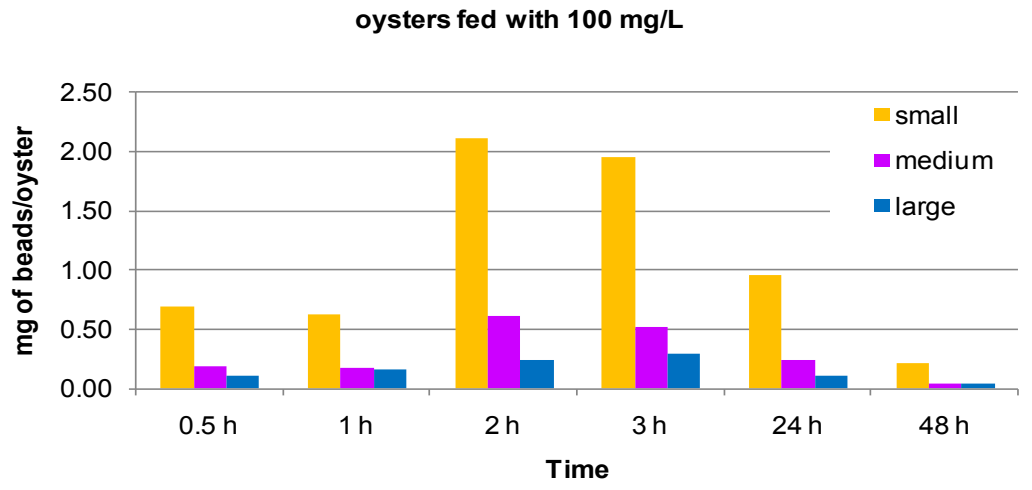

Figure 3: Concentration of fluorescent beads in the digestive gland of oysters fed with $100 \mathrm{mg} / \mathrm{L}$ of a mixture of alginate capsules of different sizes.

mussels fed with $10 \mathrm{mg} / \mathrm{L}$

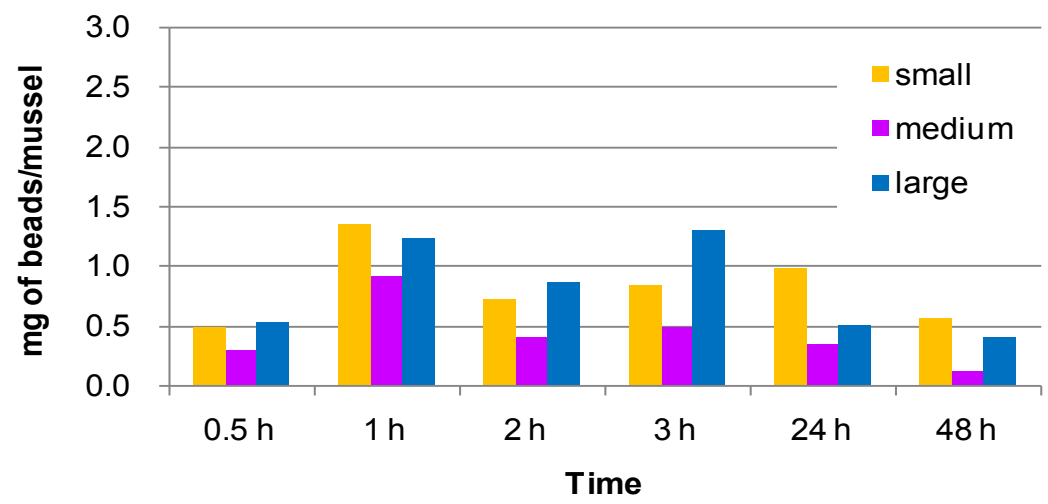

Figure 4: Concentration of fluorescent beads in the digestive gland of mussels fed with $10 \mathrm{mg} / \mathrm{L}$ of a mixture of alginate capsules of different sizes. 


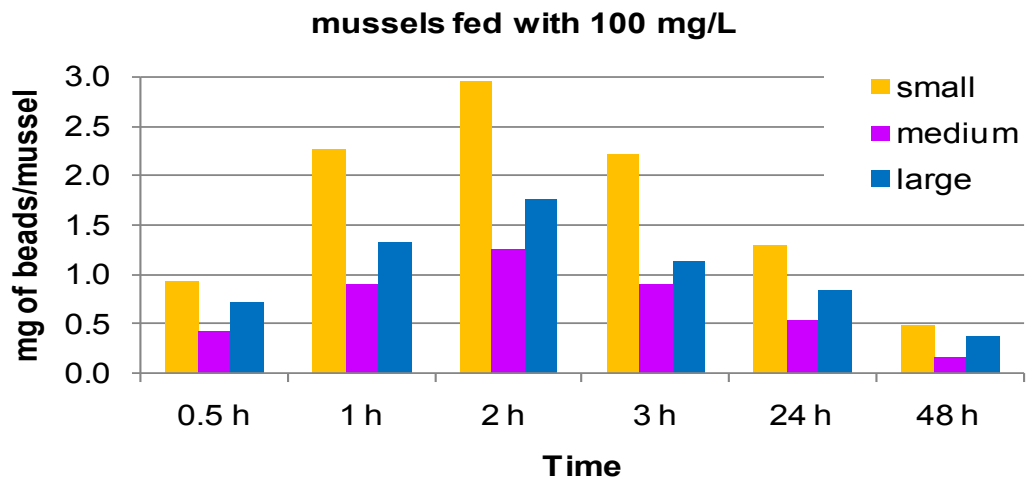

Figure 5: Concentration of fluorescent beads in the digestive gland of mussels fed with $100 \mathrm{mg} / \mathrm{L}$ of a mixture of alginate capsules of different sizes.

Mussels were more efficient than oysters at incorporating alginate beads that were observed by light microscopy as intact in the digestive gland of both shellfish during feeding period (Fig 6). Fluorescent beads of different colours embedded in alginate capsules were also observed bound to the mucus string (Fig 7) and later broken in faeces.
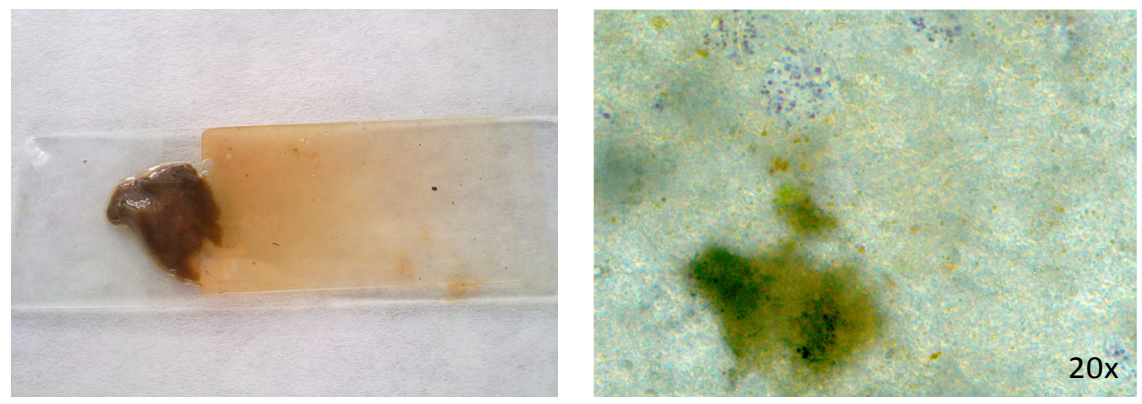

Figure 6: Alginate beads in mussel's digestive gland ( $2 \mathrm{~h}$ after feeding).

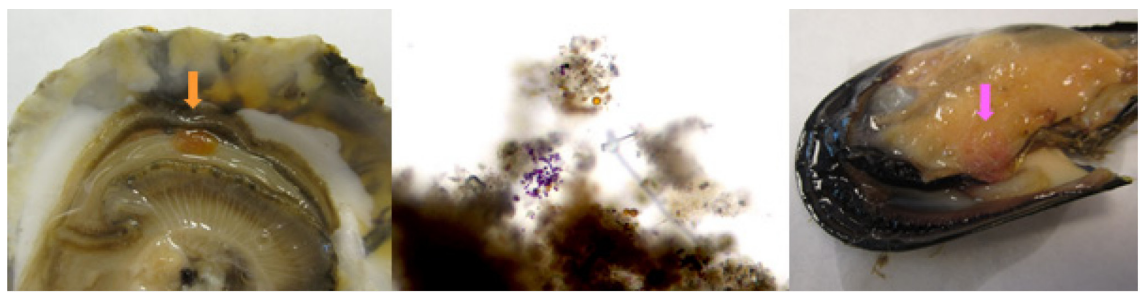

Figure 7: Fluorescent beads of different colours embedded in alginate capsules and bound to mucus string. 


\section{Discussion}

Microencapsulated marine polysaccharides are a good alternative to be applied in aquaculture for the administration of drugs or other biological compounds [4]. It has been previously demonstrated that alginates can be useful natural polymers for microencapsulation and therapeutic applications [5]. Some studies have used calcium alginate capsules for oral administration of fish probiotic bacteria [6]. Alginate has been used as a carrier for inclusion of microbeads to be introduced into shellfish due to its good mucoadhesive properties. It has been shown through the experimental work that both shellfish species tested, mussels and oysters, ingest preferentially smaller alginate capsules. To monitor the ingestion of microbeads and the later depuration we demonstrated that using only digestive glands and $100 \mathrm{mg} / \mathrm{L}$ as a feeding concentration produced satisfactory results.

The feasibility of using alginate beads to transport probiotic diets and release them into digestive organs has been demonstrated in these experiments of the BEADS project. Intact alginate beads were observed by light microscopy in the digestive gland of mussels during feeding and broken beads were found in faeces as shown in Fig 8. Therefore, during passage through the intestine of the shellfish, the contents of alginate capsules, a probiotic diet for example, are released.

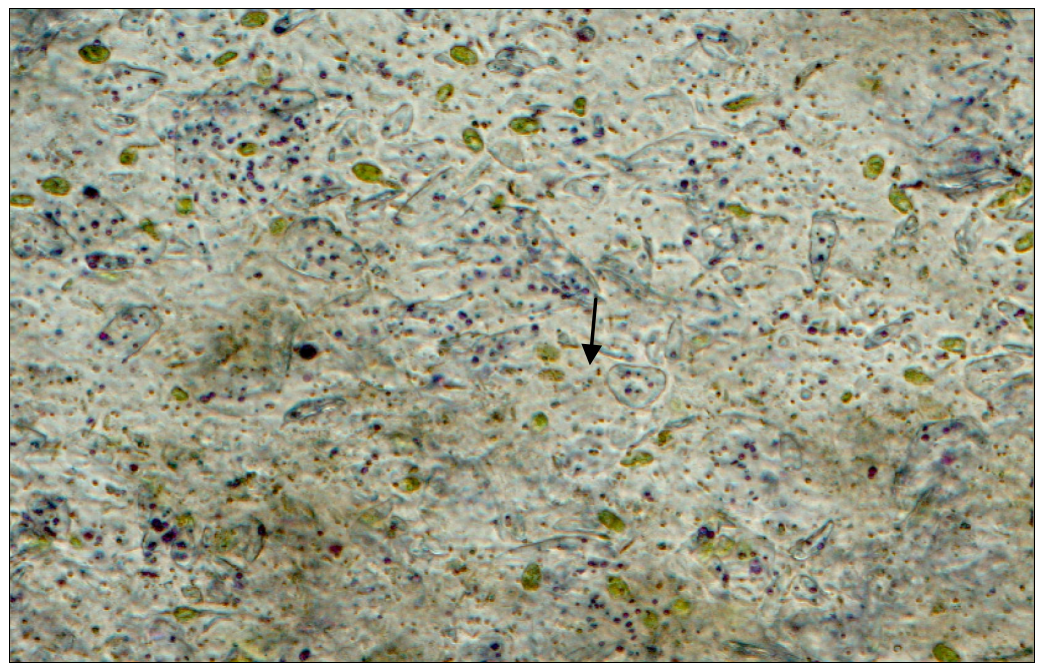

Figure 8: Alginate capsules containing non degradable fluorescent dye microbeads (arrow) in faeces of mussels.

In this study, alginate capsules were used to transport active ingredients to the digestive organs of shellfish. In conclusion, active agents embedded by alginate capsules are released during passage through the intestine, providing a useful tool to transport potential active agents against bacteria, virus, parasites or marine biotoxins. 


\section{Acknowledgement}

This work was financed by the project "BEADS", Grant Agreement Number 262649. For more information visit our website http://www. projectbeads.eu/.

\section{References}

[1] FAO, FAO Fisheries \& Aquaculture - Global Statistics Collection, 2012.

[2] New Trends in Marine and Freshwater Toxins: Food Safety concerns., ed. A.G.C.J.M. Vieites, New York: Nova Science Publishers, Inc, 2012.

[3] Lynch, S., A., M.F. Mulcahy, and S.C. Culloty, Efficiency of diagnostic techniques for the parasite, Bonamia ostreae in the flat oyster Ostrea edulis. Aquaculture, 281: p. 17-21, 2008.

[4] Borgogna, M., B. Bellich, and A. Cesàro, Marine polysaccharides in microencapsulation and application to aquaculture: "From sea to sea". Marine Drugs, 9(12): p. 2572-2604, 2011.

[5] Goh, C.H., P.W.S. Heng, and L.W. Chan, Alginates as a useful natural polymer for microencapsulation and therapeutic applications. Carbohydrate Polymers, 88(1): p. 1-12, 2011.

[6] Rosas-Ledesma, P., et al. Calcium alginate capsules for oral administration of fish probiotic bacteria: Assessment of optimal conditions for encapsulation. Aquaculture Research, 43(1): p. 106-116, 2011. 